\title{
How does wall slippage affect hydrodynamic dispersion?
}

\author{
Chiu-On Ng
}

Received: 1 April 2010/Accepted: 17 May 2010/Published online: 10 June 2010

(C) The Author(s) 2010. This article is published with open access at Springerlink.com

\begin{abstract}
How hydrodynamic dispersion is affected by wall slip remains to be fully understood. An attempt is made in this article looking into this issue for dispersion in some elementary pressure-driven flows. Both the long-time Taylor-Aris dispersion and the early-phase convectiondominated dispersion are investigated, analytically and numerically, respectively. The mean and the variance of the residence time distribution are also examined. In the basic case where the walls of a parallel-plate channel have equal slip lengths, the slip is in general to reduce the spread of a solute cloud in a finite channel by either increasing the convection speed or decreasing the dispersivity. However, the decreasing effect of slip on dispersion can be diminished or even reversed by unequal slip lengths and/or phase exchange with the wall. The convection-dominated regime is investigated, following a recently proposed transportbased method, to determine how the mean residence time and variance of elution profiles may change with axial positions depending on the slip.
\end{abstract}

Keywords Microchannel flow · Boundary slip · Taylor dispersion - Residence time distribution

\section{Introduction}

It was pointed out in many studies on slip flow in microchannels or microcapillaries that wall slippage would affect hydrodynamic dispersion (e.g. Cottin-Bizonne et al. 2004; Hendy et al. 2005; Choi et al. 2006; Adrover et al. 2009).

C.-O. $\mathrm{Ng}(\bowtie)$

Department of Mechanical Engineering, The University of Hong Kong, Pokfulam Road, Hong Kong, People's Republic of China e-mail: cong@hku.hk
The common thinking is that wall slip can reduce the crosssectional velocity gradient, and therefore, will reduce the dispersion as well. Dispersion is unwanted in separation processes, but is desirable in mixing processes. For example, axial dispersion will reduce the resolution and limit the throughput when multiple, periodically injected, samples are analyzed in a continuous-flow microfluidic system (Stone et al. 2004; Kreutzer et al. 2008). In micromixers, dispersion is, however, required in order to shorten the mixing time and to enhance the mixing quality (Wu and Nguyen 2005). Micro-chromatography, which is now widely used in micro-chemical and biological analysis, works on the principle of Taylor dispersion. It is important that one can evaluate in a more exact manner how boundary slip will affect dispersion. The answer to this question is, however, not as obvious as it appears.

Although hydrodynamic dispersion has been extensively studied in the context of microfluidics (e.g. Beard 2001; Rush et al. 2002; Ajdari et al. 2006; Dutta et al. 2006; Vikhansky 2009), the effect of boundary slip on dispersion is yet to be fully understood.

Slip on boundaries will definitely alter the velocity profile, but not necessarily reduce the velocity gradient. An example is when the flow is under a fixed pressure difference through a channel with a constant slip length. The velocity profile is only uniformly shifted by the boundary slip, while the velocity gradient remains unchanged. In other cases, asymmetrical slippage on the walls may to the contrary increase the velocity gradient.

An attempt is made in this study to examine for some elementary pressure-driven flows how boundary slip affects dispersion, both quantitatively and qualitatively. Our scope is limited to homogeneous walls with a constant slip length; walls with heterogeneous (or spatially variable) slippage are not considered here. Slip length, which 
measures the degree of slip of a surface, refers to the depth into the surface where the velocity profile would extrapolate to zero. Slip can be intrinsic as arising from hydrophobicity of a surface, or can be apparent as caused by microbubbles trapped in a rough surface (Lauga et al. 2007). For a properly micro-engineered superhydrophobic surface, the slip length can be as large as hundred microns (e.g. Choi et al. 2006; Lee et al. 2008), which is comparable to the height or radius of a microchannel (Cheng et al. 2009).

A microchannel with micropatterned walls may have the following length and velocity scales (e.g. see Rothstein 2010): size of microfeatures on the wall $d=O(0.1-$ 10) $\mu \mathrm{m}$; height $h=O(100) \mu \mathrm{m}$; slip length $\lambda=O(10-$ 100) $\mu \mathrm{m}$; length $L=O(1-10) \mathrm{cm}$; velocity $\bar{u} \geq O$ (100) $\mu \mathrm{m} / \mathrm{s}$. Therefore, it is reasonable to assume that $d \leq \lambda \sim h \ll L$. It is also reasonable to assume that the Péclet number $\mathrm{Pe}=\bar{u} h / D \geq O(10)$, where $D=O\left(10^{-9}\right) \mathrm{m}^{2} / \mathrm{s}$ is the molecular diffusivity of liquid. These assumptions form the basis of the present study. First, the microfeatures are of a size much smaller than the macroscopic length of the channel. As far as axial flow and dispersion are concerned, the microscale heterogeneities can be homogenized so that their effects show up as apparent boundary slip, and are thereby represented by an effective slip length. The slip length will be a constant if the micropattern does not vary on the macroscale. We are not concerned with flow and transport on the microscale. Of course, the Knudsen number must be so low that the fluid can be regarded as a continuum. Second, axial dispersion will be of more significance than molecular diffusion, since the ratio of dispersivity to diffusivity is proportional to the square of the Péclet number, which is large here. Third, by long-time developed dispersion (Sects. 2, 3), we mean that the transport time by axial dispersion is much longer than that by molecular diffusion across the channel section, $t \gg h^{2} / D$. In practice, the time required to attain steady-state dispersion can be, however, as short as $t \sim 0.5 h^{2} / D$ ( Ng and Rudraiah 2008). The early-phase transient dispersion (Sect. 4) therefore happens during small times, $t \leq h^{2} / D$.

Three independent problems are studied in this study. We first look into Taylor-Aris dispersion (i.e. the longtime steady-state dispersion) in Poiseuille flow through a channel of plane and circular cross-sections in the first and second problems (Sects. 2, 3), respectively. We then study in the third problem (Sect. 4) the early-phase time-dependent dispersion in flow through a circular channel. Under the influence of boundary slip, the steady-state dispersion coefficients in the first two problems are deduced analytically, while the transient dispersion in the third problem is studied numerically. To have a basis for comparison of results with the no-slip limit, we consider two possible cases. The first case is when the pressure gradient is fixed

so that the flow rate and hence the mean velocity will change depending on the slip. The second case is when the flow rate is fixed (the pressure gradient being correspondingly adjusted) so that the mean velocity is independent of the slip. Only in the second case is the velocity profile flattened by the slip. It will be seen that the slip effects can be qualitatively different in these two cases.

In the first problem, the two walls of the plane channel are allowed to have different slip lengths. The question here is, if one of the slip lengths is given, what is the value of the other slip length that will give rise to the smallest dispersivity? In the second problem, the combined effects due to slip and phase exchange are examined. It is shown how the mass partitioning and exchange between fluid and wall may diminish the decreasing effect, or even to the contrary enhance the increasing effect of slip on dispersion. The variance of the residence time distribution, which reflects the extent of dispersive mixing in a channel, is also expressed in terms of the slip length and other parameters. In the third problem, the effect of slip on the elution of a finite solute cloud as a function of axial position is first discussed. We then follow a recent study by Adrover et al. (2009) on examining the possible occurrence of saturation of the first and second moments of elution profiles at very short axial distances, where dispersion is dominated by convection.

\section{Taylor-Aris dispersion in parallel-plate channel}

We first consider long-time dispersion in steady pressuredriven flow through an infinitely extended parallel-plate channel of height $h$; see Fig. 1a. We assume that, for generality, the two walls have different slip lengths denoted by $\lambda_{1}$ and $\lambda_{2}$. Based on the coordinate $y$ shown in Fig. 1a, the velocity profile can be readily found to be

$u(\hat{y})=\frac{K h^{2}}{2 \mu}\left[-\hat{y}^{2}+\left(\frac{1+2 \hat{\lambda}_{2}}{1+\hat{\lambda}_{1}+\hat{\lambda}_{2}}\right)\left(\hat{y}+\hat{\lambda}_{1}\right)\right]$,

where $K=-\mathrm{d} p / \mathrm{d} x$ is the axial pressure gradient, $\mu$ is the dynamic viscosity of the fluid, and $\left(\hat{y}_{1}, \hat{\lambda}_{1}, \hat{\lambda}_{2}\right)=$

(a)

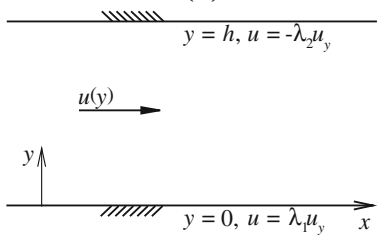

Fig. 1 Definition sketches for pressure-driven slip flow through a channel of a parallel-plate cross section, and $\mathbf{b}$ circular cross section. In a, the two plane walls at $y=0$ and $y=h$ have constant slip lengths $\lambda_{1}$ and $\lambda_{2}$, respectively. In $\mathbf{b}$, the cylindrical wall at $r=a$ has a constant slip length $\lambda$ 
$\left(y, \lambda_{1}, \lambda_{2}\right) / h$ are the normalized coordinate and slip lengths. The section-mean velocity is then given by

$\bar{u}=\int_{0}^{1} u \mathrm{~d} \hat{y}=\frac{K h^{2}}{2 \mu}\left[\frac{\left(1+2 \hat{\lambda}_{1}\right)\left(1+2 \hat{\lambda}_{2}\right)}{2\left(1+\hat{\lambda}_{1}+\hat{\lambda}_{2}\right)}-\frac{1}{3}\right]$.

The Taylor dispersion coefficient is given by (Mei et al. 1996):

$D_{T}=\bar{N} \bar{u}-\overline{N u}$

where the overbar denotes section averaging. The function $N(y)$ is governed by the boundary-value problem:

$D \frac{\mathrm{d}^{2} N}{\mathrm{~d} y^{2}}=u-\bar{u} \quad$ in $0<y<h$,

$D \frac{\mathrm{d} N}{\mathrm{~d} y}=0 \quad$ at $y=0, h$

where $D$ is the molecular diffusivity. A uniqueness condition, such as $\bar{N}=0$, can be specified in order to solve for $N$ uniquely. This condition, however, has no effect on the dispersion coefficient given in Eq. 3. After some algebra solving the problem above, the dispersion coefficient can be determined as follows:

$D_{T}=\left[\frac{8}{945}-\frac{\left(1+2 \hat{\lambda}_{1}\right)\left(1+2 \hat{\lambda}_{2}\right)}{120\left(1+\hat{\lambda}_{1}+\hat{\lambda}_{2}\right)^{2}}\right] \frac{K^{2} h^{6}}{4 \mu^{2} D}$,

which, on substituting Eq. 2, can alternatively be written as

$D_{T}=\frac{\left[\frac{32}{105}\left(1+\hat{\lambda}_{1}+\hat{\lambda}_{2}\right)^{2}-\frac{3}{10}\left(1+2 \hat{\lambda}_{1}\right)\left(1+2 \hat{\lambda}_{2}\right)\right]}{\left[1+4\left(\hat{\lambda}_{1}+\hat{\lambda}_{2}\right)+12 \hat{\lambda}_{1} \hat{\lambda}_{2}\right]^{2}} \frac{\bar{u}^{2} h^{2}}{D}$.

In the particular case when the two slip lengths are equal to each other, $\hat{\lambda}_{1}=\hat{\lambda}_{2}=\hat{\lambda}$, the two expressions above are respectively simplified to

$D_{T}=\frac{K^{2} h^{6}}{30240 \mu^{2} D}=\frac{\bar{u}^{2} h^{2}}{210(1+6 \hat{\lambda})^{2} D}$.

Hence, when both walls are non-slip $(\hat{\lambda}=0)$, the classical result (Wooding 1960) for dispersivity in a plane channel is recovered:

$D_{T 0}=\frac{\bar{u}_{0}^{2} h^{2}}{210 D}, \quad$ where $\quad \bar{u}_{0}=\frac{K h^{2}}{12 \mu}$ for $\hat{\lambda}_{1}=\hat{\lambda}_{2}=0$.

The slip effects are to be seen through comparison with the no-slip limit. In this regard, we need to consider two cases separately. The first case is when the flow is controlled by pressure gradient so that $K$ is kept fixed, and hence the expression in Eq. 6 should be used. In this case, the mean velocity $\bar{u}$ will increase with the slip lengths according to
Eq. 2. The second case is when the flow is controlled by flow-rate so that $\bar{u}$ is kept fixed, and hence the expression in Eq. 7 should be used. In this case, the mean velocity is invariant, but the velocity profile is flattened by the slip.

When the flow is pressure-gradient controlled, the dispersion coefficient given in Eq. 6 turns out to be the minimum when the two slip lengths are equal to each other: $\hat{\lambda}_{1}=\hat{\lambda}_{2}$, by which

$\min D_{T}=D_{T 0} \quad$ (under fixed $\left.\mathrm{K}\right)$,

where $D_{T 0}$ is the no-slip dispersion coefficient, as given in Eq. 9. It means that for a channel with equal slip lengths, the boundary slip will have no effect at all on the dispersion coefficient, when under the same pressure gradient. This is understandable since in this particular case the velocity profile is shifted uniformly by the slip velocity; the velocity gradient remains unchanged. This amounts to a uniform flow being added to the Poiseuille flow. The same slip term is added to the velocity profile and the mean velocity. Hence, the velocity deviation from the mean, $u-\bar{u}$, which drives the dispersion, is independent of the slip length. In general, when the two slip lengths are disparate, the dispersion coefficient can only be larger than the no-slip limit. Disparity in slip lengths implies one boundary slip being larger than the other, and hence the velocity profile becomes asymmetrical about the channel centerline. This amounts to a Couette flow being added to the Poiseuille flow, thereby increasing the dispersion coefficient. Figure 2 shows the normalized dispersion coefficient $\hat{D}_{T}=D_{T} / D_{T 0}$ as a function of $\hat{\lambda}_{1}$ and $\hat{\lambda}_{2}$ according to Eq. 6 . The

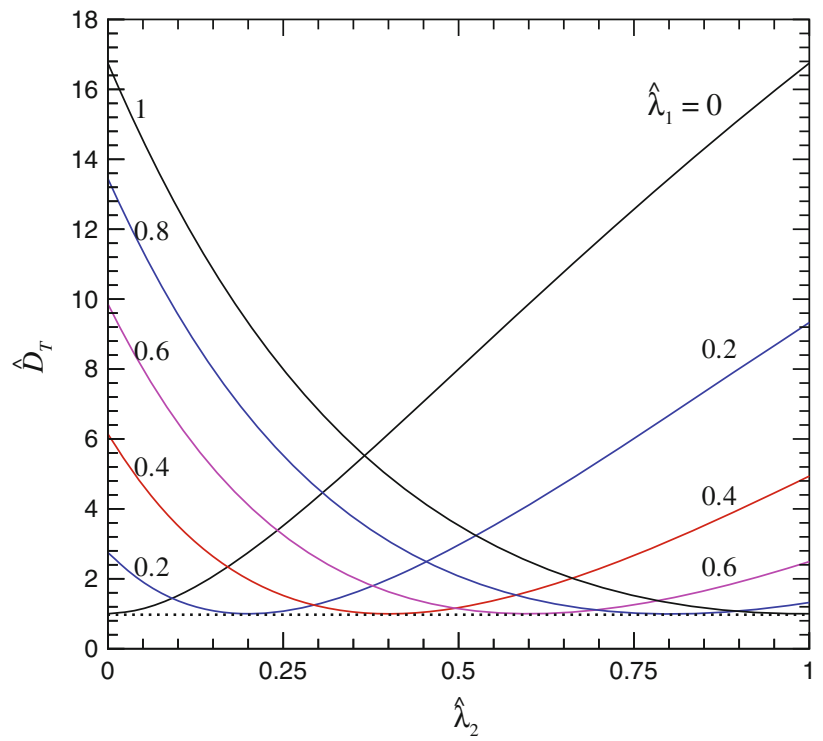

Fig. 2 The normalized dispersion coefficient $\hat{D}_{T}=D_{T} / D_{T 0}$ as a function of the two slip lengths $\hat{\lambda}_{1}$ and $\hat{\lambda}_{2}$, as given by Eq. 6 for flow through a parallel-plate channel under fixed pressure gradient. The dotted line is the no-slip limit $\hat{D}_{T}=1$ 
dispersion coefficient reaches its minimum when $\hat{\lambda}_{1}=\hat{\lambda}_{2}$, and can be many times larger than the minimum when the two slip lengths are very different from each other. In this case, the boundary slip will enhance the convection speed, but will not decrease the dispersivity.

The slip effect becomes different when it is flow-rate controlled. For a given value of $\hat{\lambda}_{1}$, the dispersion coefficient in Eq. 7 will attain the minimum value given by

$\min D_{T}=\frac{\bar{u}^{2} h^{2}}{240\left(32 \hat{\lambda}_{1}^{2}+11 \hat{\lambda}_{1}+1\right) D}<D_{T 0}$

(under fixed $\bar{u}$ )

when

$\hat{\lambda}_{2}=\frac{128 \hat{\lambda}_{1}^{2}+24 \hat{\lambda}_{1}+1}{124 \hat{\lambda}_{1}+20}>\hat{\lambda}_{1}$.

The minimum value, which is a monotonically decreasing function of $\hat{\lambda}_{1}$, happens when $\hat{\lambda}_{2}$ is slightly larger than (N.B., not exactly equal to) the given $\hat{\lambda}_{1}$. (Of course, if the given condition is $\hat{\lambda}_{1}+\hat{\lambda}_{2}=$ constant, the minimum value will happen when $\hat{\lambda}_{1}=\hat{\lambda}_{2}$ by symmetry.) Figure 3 shows the normalized dispersion coefficient $\hat{D}_{T}=D_{T} / D_{T 0}$ as a function of $\hat{\lambda}_{1}$ and $\hat{\lambda}_{2}$ according to Eq. 7. The dispersion coefficient is smaller than the no-slip limit as long as it is in close proximity to the minimum given by Eq. 11. In fact, the dispersion coefficient will be substantially decreased when the two slip lengths are both large and kept close to each other. In contrast, it will be substantially increased when one wall is non-slip and the other wall has strong slip.

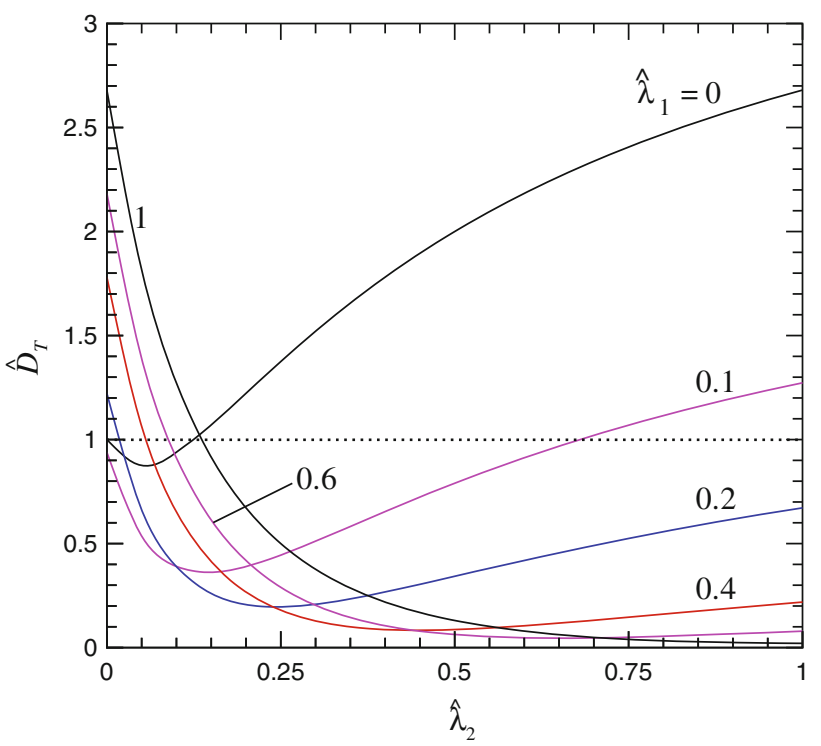

Fig. 3 The normalized dispersion coefficient $\hat{D}_{T}=D_{T} / D_{T 0}$ as a function of the two slip lengths $\hat{\lambda}_{1}$ and $\hat{\lambda}_{2}$, as given by Eq. 7 for flow through a parallel-plate channel under fixed flow rate. The dotted line is the no-slip limit $\hat{D}_{T}=1$
A larger difference in slip of the two walls will result in a more skewed or nonuniform velocity profile, and hence a larger dispersion coefficient. In this case, the boundary slip is not to change the convection speed, but will increase or decrease the spreading rate depending on the slip lengths.

While the rate of broadening of a solute cloud about its center is given by the dispersion coefficient, the extent of dispersive mixing in a reactor is reflected by the residence time distribution (RTD), which depends on both convection and dispersion. The RTD, also called the exit age distribution curve (Levenspiel 1999), is a normalized distribution of the concentration measured at the exit of a vessel as a function of time. For a pulse input into a channel of length $L$, the RTD is

$E(t)=\frac{C(L, t)}{\int_{0}^{\infty} C(L, t) \mathrm{d} t}$

where $C(L, t)$ is the solute concentration at the outlet of the channel. The $E$ curve, the area under which is unity, is characterized by the mean residence time $t_{c}$ and variance $\sigma^{2}$ :

$t_{c}=\int_{0}^{\infty} t E \mathrm{~d} t, \quad \sigma^{2}=\int_{0}^{\infty} t^{2} E \mathrm{~d} t-t_{c}^{2}$.

In terms of normalized time $\theta=t / t_{c}$, the nondimensional RTD and variance can be defined as $E_{\theta}=t_{c} E$ and $\sigma_{\theta}^{2}=$ $\sigma^{2} / t_{c}^{2}=\int_{0}^{\infty} \theta^{2} E_{\theta} \mathrm{d} \theta-1$. For a sufficiently long channel and an inert species, the mean residence time is equal to the convection time through the channel $t_{c}=L / \bar{u}$. If further assuming a Gaussian RTD, one can get (Levenspiel 1999)

$\frac{\sigma_{\theta}^{2}}{2}=\frac{D_{T}}{\bar{u} L}=\frac{D_{T}}{\bar{u}^{2} t_{c}}$,

where $D_{T} / \bar{u} L$ is known as the vessel dispersion number, which measures the extent of axial dispersion in a reactor. Based on the velocity and dispersion coefficients deduced above, and considering only the case $\hat{\lambda}_{1}=\hat{\lambda}_{2}=\hat{\lambda}$, we can obtain the following expressions for the variance under different conditions. When $K$ is fixed,

$\frac{\sigma_{\theta}^{2}}{2}=\left\{\begin{array}{cl}K h^{4} /[2520(1+6 \hat{\lambda}) \mu D L] & \text { for a channel of fixed } L \\ h^{2} /\left[210(1+6 \hat{\lambda})^{2} D t_{c}\right] & \text { for a channel of fixed } t_{c}\end{array}\right.$.

When $\bar{u}$ is fixed,

$\frac{\sigma_{\theta}^{2}}{2}=\frac{\bar{u} h^{2}}{210(1+6 \hat{\lambda})^{2} D L}=\frac{h^{2}}{210(1+6 \hat{\lambda})^{2} D t_{c}}$.

It is apparent that, through the factor $(1+6 \hat{\lambda})$ or $(1+6 \hat{\lambda})^{2}$ in the denominator of the expressions above, the boundary slip will indeed reduce the variance of the RTD whether the 
flow is pressure-gradient or flow-rate controlled. We recall that, in the case of fixed $K, D_{T}$ is independent of $\hat{\lambda}$, but $\bar{u}$ increases according to $(1+6 \hat{\lambda})$. This explains why the vessel dispersion number will decrease according to $(1+$ $6 \hat{\lambda})^{-1}$ for a channel of fixed length. On the other hand, for a channel of fixed mean residence time, the length also increases according to $(1+6 \hat{\lambda})$, and therefore, the vessel dispersion number will decrease according to $(1+6 \hat{\lambda})^{-2}$. In the latter case, the same expression is applicable whether $K$ or $\bar{u}$ is fixed.

\section{Taylor-Aris dispersion in circular channel}

We next consider Taylor-Aris dispersion in steady Poiseuille flow through an infinitely long circular channel of radius $a$; see Fig. 1b. For axisymmetry, a constant slip length $\lambda$ is assumed. The velocity profile can readily be found to be

$u(\hat{r})=\beta \bar{u}\left(1-\hat{r}^{2}+2 \hat{\lambda}\right)$,

where $(\hat{r}, \hat{\lambda})=(r, \lambda) / a$ are the normalized radial coordinate and slip length,

$\beta=\frac{2}{1+4 \hat{\lambda}}$,

and

$\bar{u}=\frac{K a^{2}}{8 \mu}(1+4 \hat{\lambda})$

is the section-mean velocity, in which $K=-\mathrm{d} p / \mathrm{d} z$ is the axial pressure gradient, and $\mu$ is the dynamic viscosity of the fluid.

To make the present problem more interesting, we consider also that the channel wall is coated with a thin retentive layer so that there is a kinetic reaction of phase exchange between the fluid and the wall. While the fluid phase is mobile, the wall phase is immobile; the phase exchange is reversible and the rate of change is controlled by the departure from local equilibrium of the two phases. Dispersion under such a partition effect is of relevance in chromatography, and has been studied by Aris (1959), and many others. Following the model by $\mathrm{Ng}(2000,2006)$ and $\mathrm{Ng}$ and Yip (2001), we assume that the phase exchange is describable by a first-order kinetic relationship. A formal expression for the Taylor dispersion coefficient has been deduced by Ng (2006):

$D_{T}=\frac{1}{R} \overline{N\left(\frac{\bar{u}}{R}-u\right)}+2 N_{s} \frac{\bar{u}}{a R^{2}}$,

where the overbar denotes section averaging, and $R=1+2 \alpha / a$ is the retardation factor, in which $\alpha$ is the equilibrium partition ratio of the wall phase concentration (mass sorbed on unit area of wall) to the fluid phase concentration (mass in unit volume of fluid). The function $N(r)$ and the term $N_{s}$ are governed by the following boundary-value problem:

$\frac{D}{r} \frac{\mathrm{d}}{\mathrm{d} r}\left(r \frac{\mathrm{dN}}{\mathrm{d} r}\right)=u-\frac{\bar{u}}{R} \quad$ in $0<r<a$,

$-D \frac{\mathrm{dN}}{\mathrm{d} r}=-\alpha \frac{\bar{u}}{R}=k\left(\alpha N-N_{s}\right), \quad$ at $r=a$,

where $D$ is the molecular diffusivity, and $k$ is the reaction rate constant for the phase exchange.

After some algebra solving the problem above, the dispersion coefficient that is under the combined influence of boundary slip and phase exchange is found to be:

$D_{T}=F \frac{\bar{u}^{2} a^{2}}{R D}+\frac{2 \alpha}{k} \frac{\bar{u}^{2}}{a R^{3}}$,

where

$F=\frac{11 \beta^{2}}{192}-\frac{\beta}{6 R}+\frac{1}{8 R^{2}}+\beta \hat{\lambda}\left(-\frac{1}{2 R}+\frac{\beta}{3}+\frac{\beta \hat{\lambda}}{2}\right)$.

The last term on the right-hand side of Eq. 24 is a component due to the kinetic mass transfer between the wall and fluid phases; it is not affected by the slip. In the absence of boundary slip, $\hat{\lambda}=0$ and $\beta=2$, the no-slip limit of the coefficient ( $\mathrm{Ng}$ 2006) can be recovered:

$D_{T 0}=F_{0} \frac{\bar{u}_{0}^{2} a^{2}}{R D}+\frac{2 \alpha}{k} \frac{\bar{u}_{0}^{2}}{a R^{3}}$,

where $\bar{u}_{0}=K a^{2} / 8 \mu$, and

$F_{0}=\frac{11}{48}-\frac{1}{3 R}+\frac{1}{8 R^{2}}$.

Further in the absence of wall retention, $\alpha=0$ and $R=1$, the coefficient above reduces to the classical Taylor dispersion coefficient (Taylor 1953): $D_{T 0}^{*}=\bar{u}_{0}^{2} a^{2} / 48 D$.

If instead there is wall slip $(\hat{\lambda}>0)$ but no wall retention $(\alpha=0)$, the coefficient in Eq. 24 can be simplified to

$D_{T}^{\prime}=\frac{\bar{u}^{2} a^{2}}{48(1+4 \hat{\lambda})^{2} D}$.

The section-mean velocity $\bar{u}=(1+4 \hat{\lambda}) \bar{u}_{0}$ when the pressure gradient is fixed, or $\bar{u}=\bar{u}_{0}$ when the flow rate is fixed. In the former case, $D_{T}^{\prime}=D_{T 0}^{*}$, or the dispersion coefficient is independent of the slip length. In the latter case, the dispersion coefficient decreases with increasing slip length according to the factor $(1+4 \hat{\lambda})^{-2}$.

Let us from here on assume that the phase exchange is so fast (i.e. $k \rightarrow \infty$ ) that local equilibrium prevails in the partitioning. Hence, on dropping the last term in Eqs. 24 and 26 , we define a normalized dispersion coefficient 
$\hat{D}_{T}=\frac{D_{T}}{D_{T 0}}=\left(\frac{\bar{u}}{\bar{u}_{0}}\right)^{2}\left(\frac{F}{F_{0}}\right)$.

Again, to facilitate comparison with the no-slip limit, we distinguish between the two cases when either the pressure gradient or the flow rate is fixed. Figures 4 and 5 show the dependence of $\hat{D}_{T}$ on the dimensionless partition parameter $\hat{\alpha}=\alpha / a$ and the dimensionless slip length $\hat{\lambda}$ for these two cases, respectively.

When under fixed pressure gradient (Fig. 4), the dispersion coefficient is unaffected by the slip when there is no wall retention, as analytically shown above. As explained in the previous section, uniform wall slippage in this case will not alter the velocity gradient, and therefore, has no effect on the dispersion coefficient. In the presence of even a small degree of wall retention, the dispersion coefficient can be, however, materially enhanced by the boundary slip. This is because the wall phase, unlike the mobile fluid phase, does not experience the boundary slip. Mass retained on the wall will be released back to the flow only at times subsequent to the passing of the solute cloud peak. This leads to tailing in the axial concentration distribution. Increasing the slip will effectively lengthen the tailing, thereby enhancing the dispersion.

When under fixed flow rate (Fig. 5), the dispersion coefficient decreases as the boundary slip increases, obviously because of a more flattened velocity profile. As analytically shown above, the coefficient decreases according to the factor $(1+4 \hat{\lambda})^{-2}$ for a non-reactive species. The phase partitioning, if present, will counteract the

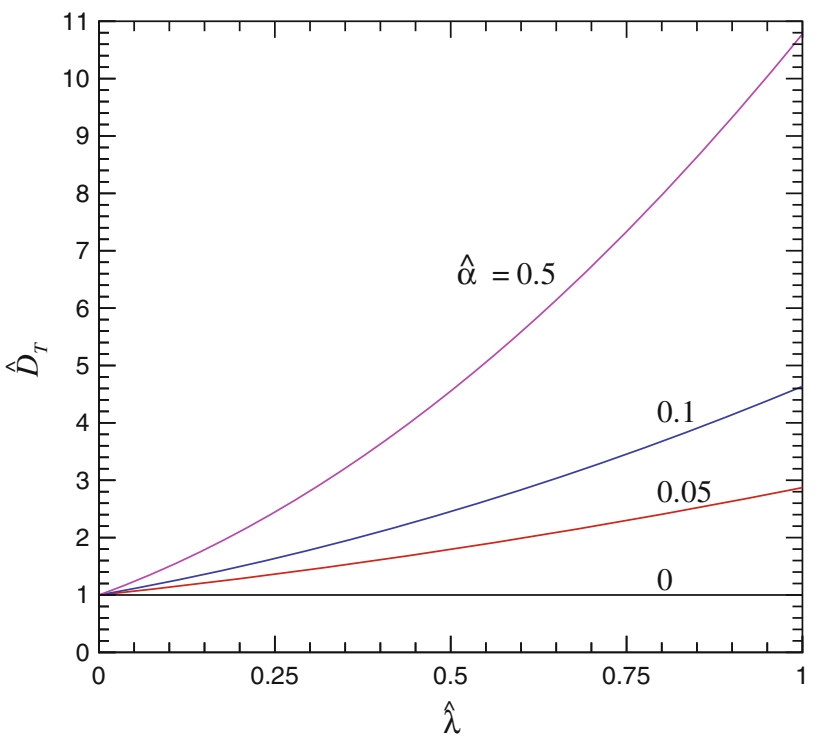

Fig. 4 The normalized dispersion coefficient $\hat{D}_{T}=D_{T} / D_{T 0}$ as a function of the dimensionless partition parameter $\hat{\alpha}=\alpha / a$ and the slip length $\hat{\lambda}$, as given by Eq. 29 for flow through a circular channel under fixed pressure gradient. Note that $\hat{D}_{T}=1$ is the no-slip limit

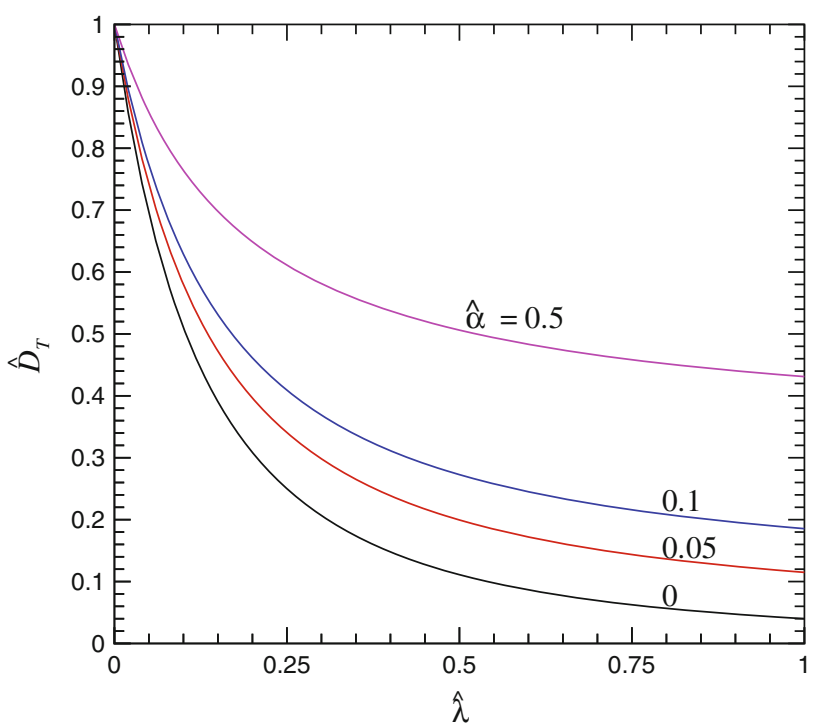

Fig. 5 The normalized dispersion coefficient $\hat{D}_{T}=D_{T} / D_{T 0}$ as a function of the dimensionless partition parameter $\hat{\alpha}=\alpha / a$ and the slip length $\hat{\lambda}$, as given by Eq. 29 for flow through a circular channel under fixed flow rate. Note that $\hat{D}_{T}=1$ is the no-slip limit

decreasing effect of slip on dispersion. The larger the wall retention, the less the dispersion coefficient is affected by the slip.

Owing to phase partition, the effective convection speed is $\bar{u} / R$. Hence, the vessel dispersion number in this case is $D_{T} R / \bar{u} L$. On substituting the dispersion coefficient deduced above, we get

$\frac{D_{T} R}{\bar{u} L}=\left\{\begin{array}{ll}G \frac{K a^{4}}{\mu D L} & \text { for fixed } K \\ F \frac{\bar{u} a^{2}}{D L} & \text { for fixed } \bar{u}\end{array}\right.$,

where $G=(1+4 \hat{\lambda}) F / 8$, and $F$ is given in Eq. 25 . The two dimensionless prefactors, $G$ and $F$, of the vessel dispersion number for flow under fixed $K$ or $\bar{u}$ are shown in Fig. 6 as functions of $\hat{\lambda}$ and $\hat{\alpha}$. In the absence of wall retention, $\hat{\alpha}=0, G$ and $F$ decrease according to $(1+4 \hat{\lambda})^{-1}$ and $(1+$ $4 \hat{\lambda})^{-2}$, respectively. The decreasing effect of wall slip will be, however, reduced by the presence of wall retention. In the case of fixed $K$, the effect will be reversed (i.e. the slip is to increase the dispersion number) for sufficiently large $\hat{\alpha}$.

\section{Transient dispersion in circular channel}

We next examine effects of slip on the transient dispersion in steady flow through a circular channel. Dispersion of a solute after injection into fluid evolves through several phases (Golay and Atwood 1979) on approaching the longtime Taylor-Aris limit. During the early phases, the transport is largely kinematic, and the solute spread is 
Fig. 6 The numerical prefactors, $G$ and $F$, of the vessel dispersion number given in Eq. 30 for flow through a circular cylinder under a fixed pressure gradient, or $\mathbf{b}$ fixed flow rate, respectively (a)

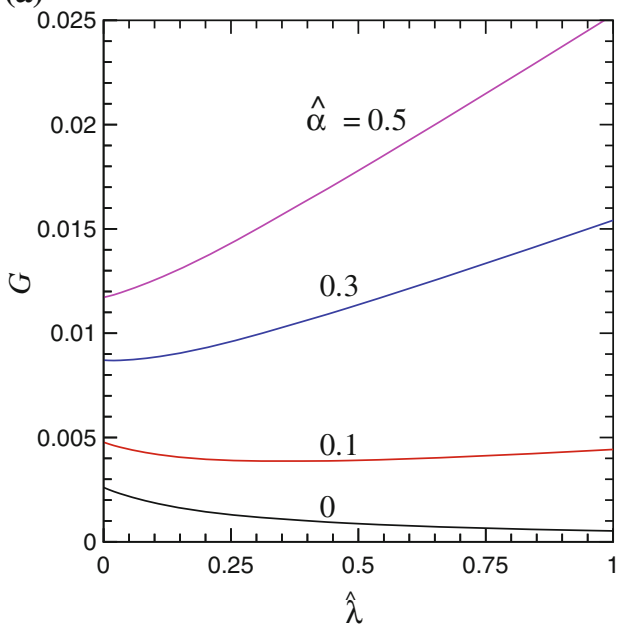

(b)

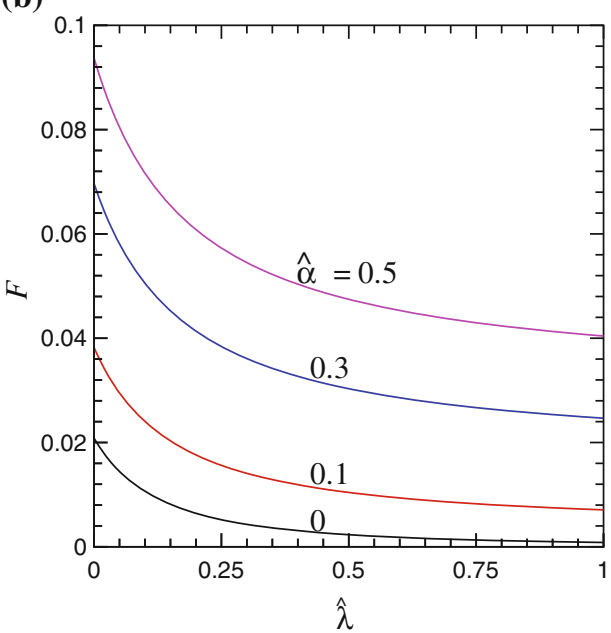

dominated by convection. Convection-dominated dispersion is important in wide-bore chromatography, as has been recently studied by Adrover et al. (2009) who proposed a transport-based approach to assess the occurrence of slip flows in a microchannel.

Ignoring wall retention, we consider the following initial-boundary-value transport problem:

$\frac{\partial \hat{C}}{\partial \hat{t}}+\hat{u} \frac{\partial \hat{C}}{\partial \hat{z}}=\frac{1}{\hat{r}} \frac{\partial}{\partial \hat{r}}\left(\hat{r} \frac{\partial \hat{C}}{\partial \hat{r}}\right)+\frac{1}{\mathrm{Pe}^{2}} \frac{\partial^{2} \hat{C}}{\partial \hat{z}^{2}} \quad$ in $0<\hat{r}<1$,

$\frac{\partial \hat{C}}{\partial \hat{r}}=0 \quad$ at $\hat{r}=0,1$,

$\hat{C}=0 \quad$ at $\hat{z} \rightarrow \pm \infty$,

$\hat{C}=\left\{\begin{array}{ll}1, & |\hat{z}| \leq \hat{z}_{s} \\ 0, & |\hat{z}|>\hat{z}_{s}\end{array} \quad \hat{t}=0\right.$,

where $\hat{C}(\hat{z}, \hat{r}, \hat{t})$ is the nondimensional concentration of a solute, $\hat{z}=z /\left(a^{2} \bar{u} / D\right)$ and $\hat{t}=t /\left(a^{2} / D\right)$ are the nondimensional axial coordinate and time, and $\mathrm{Pe}=\bar{u} a / D$ is the Péclet number. By Eqs. 18-20, the normalized velocity is $\hat{u}(\hat{r})=u / \bar{u}=2\left(1-\hat{r}^{2}+2 \hat{\lambda}\right) /(1+4 \hat{\lambda})$. Here, we consider only the case in which the flow rate is fixed so that the mean velocity $\bar{u}$ is independent of the slip. The initial input is a narrow uniform slug of length $2 z_{s}$ centered at the axial origin, which approximates to a pulse input.

We solved this problem numerically using a previously developed finite-volume code ( $\mathrm{Ng}$ and Rudraiah 2008). The numerical scheme is based on an updated version of the flux-corrected transport (FCT) algorithm, by the name of LCPFCT (Boris et al. 1993). FCT is known to be a highorder, monotone, conservative, and positivity preserving algorithm. It is capable of resolving steep gradients with an accuracy comparable to grid scale numerical resolution. The FCT scheme follows a predictor-corrector type of approach, ensuring positivity while introducing no new maxima or minima due to numerical errors in the convection process. Numerical simulation of solute dispersion using FCT was first performed by Mayock et al. (1980). Further details on how we applied this numerical scheme can be found in $\mathrm{Ng}$ and Rudraiah (2008). We note on passing that a review of the state-of-the-art computational strategies for micro and nanofluid dynamics, in the framework of molecular dynamics and continuum fluid dynamics methods, has been presented by Kalweit and Drikakis (2008).

In this study, the discretization and other input parameters used for the simulation are $\Delta \hat{t}=10^{-4}$ (or smaller for very early times), $\Delta \hat{z}=10^{-3}, \Delta \hat{r}=0.025, \hat{z}_{s}=0.002$, and $\mathrm{Pe}=1000$. The time step is small enough for the Courant number stability condition to be always satisfied. In our simulations, the total mass of the species in the system was monitored, and typically mass conservation is satisfied to an accuracy of $10^{-3}$. We used a relatively large value of the Péclet number in order to minimize the axial spreading due to molecular diffusion so that we can interpret the results purely in terms of dispersion. In fact, the Péclet number will have little effect on our results as long as it is greater than order unity. In the following, let us compare the results for three values of slip length: $\hat{\lambda}=0,0.05,0.5$.

Breakthrough/elution curves (equivalent to chromatograms that show the passing of a solute cloud through a cross section, typically the outlet of a column) based on the area-mean concentration $\hat{\hat{C}}(\hat{t})=2 \int_{0}^{1} \hat{C} \hat{r} \mathrm{~d} \hat{r}$ are shown in Fig. 7 for four axial positions, ranging from a short distance, $\hat{z}=0.0205$, where the spread is dominated by convection, to a relatively long distance, $\hat{z}=0.5005$, where the spread is affected by both convection and diffusion. We have also examined the corresponding curves based on the convected-mean (or mixing-cup average) concentration $\tilde{\hat{C}}=2 \int_{0}^{1} \hat{u} \hat{C} \hat{r} \mathrm{~d} \hat{r}$. They display similar trends to those based 
Fig. 7 Breakthrough/elution curves based on the area-mean concentration $\hat{\hat{C}}(\hat{t})=2 \int_{0}^{1} \hat{C} \hat{r} \mathrm{~d} \hat{r}$ for the early development of dispersion in a circular channel, at the axial positions $\mathbf{a} \hat{z}=$ $0.0205, \mathbf{b} \hat{z}=0.1005, \mathbf{c} \hat{z}=$ 0.2005 , and $\mathbf{d} \hat{z}=0.5005$, where the slip length $\hat{\lambda}=0,0.05,0.5$
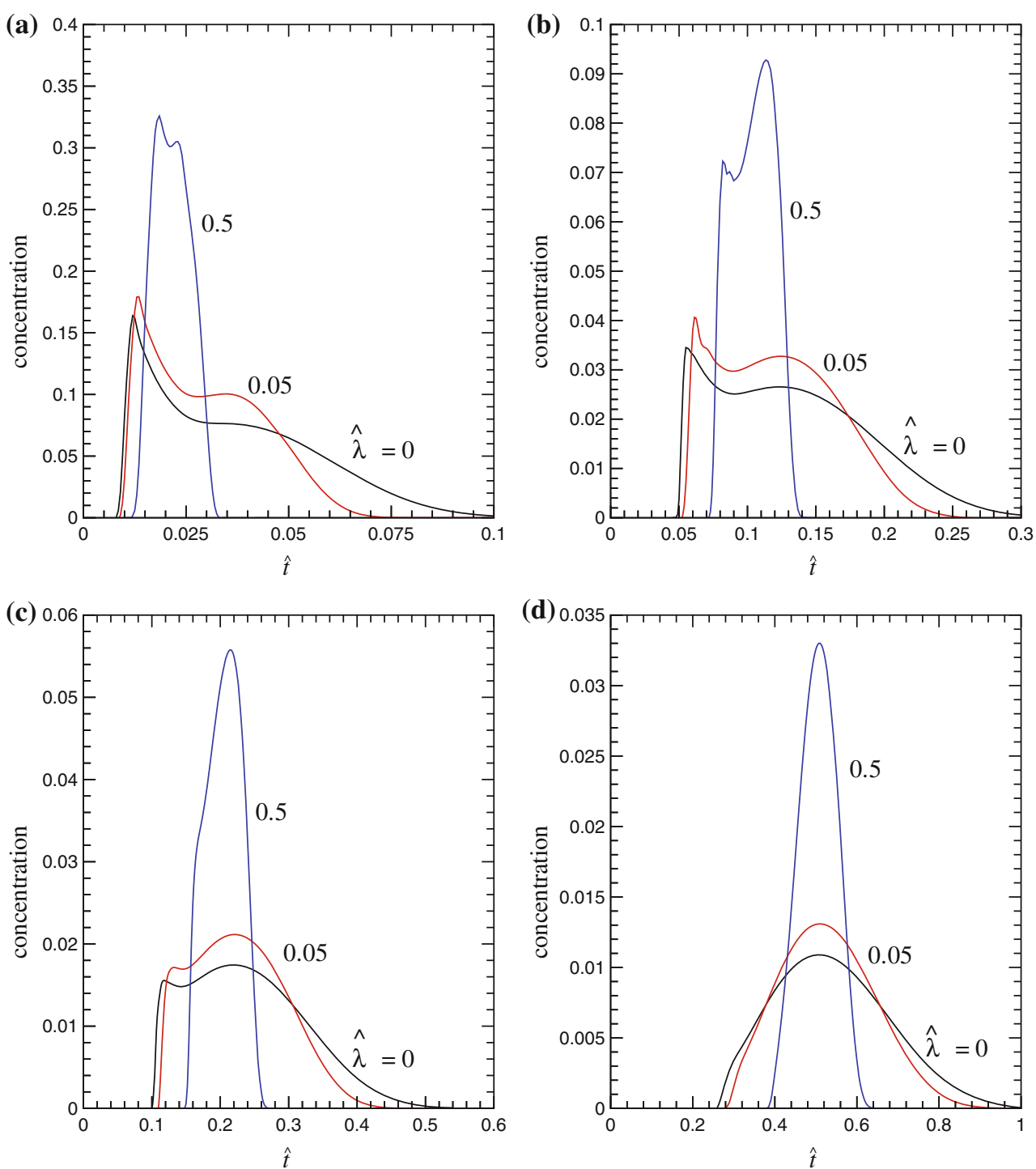

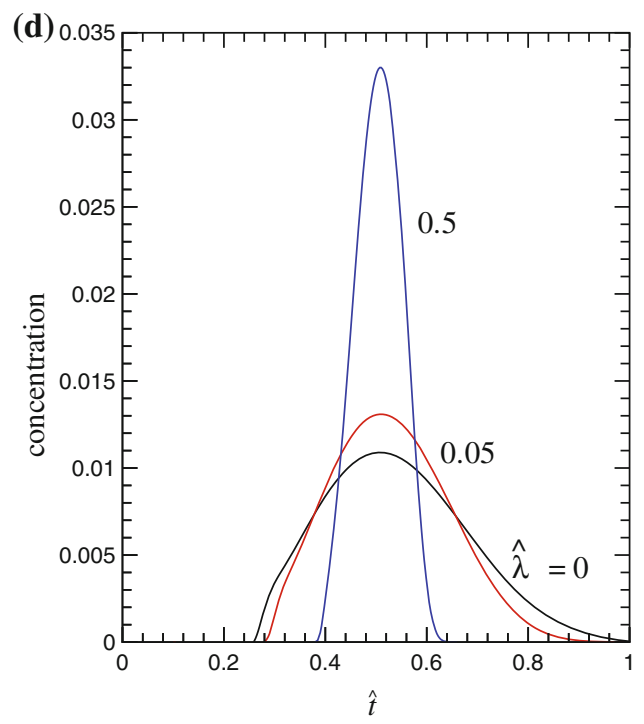

on the area-mean concentration, and therefore, are not presented here for simplicity.

Let us first review the breakthrough/elution curves corresponding to the no-slip case, which are classical, as have been reported before by, e.g. Mayock et al. (1980) Whether the dispersion is dominated by convection can be judged from the modal abscissa (i.e. the time of the peak) of a breakthrough curve (Adrover et al. 2009). At a short distance from the injection point, where convection dominates the spread, the curve will be strongly asymmetric, exhibiting a peak upon its initial sharp rise, followed by a gradual decline first according to the kinematic limit, and then branching out from this limit because of radial diffusion; see Fig. 7a. By the kinematic limit (i.e. purely convection), the front concentration at the center of the channel first arrives, at a time given by the distance divided by the centerline convection speed (which is twice the mean velocity in the no-slip case). The concentration then decreases according to $\hat{t}^{-2}$, and the curve has in theory an infinitely long tail. One can perceive that, in the absence of diffusion, the solute that sticks to the wall, because of no slip, takes an infinitely long time to be completely eluted downstream. Of course, diffusion can be ignored only up to a certain point in time, depending on the Péclet number. As the near-wall solute diffuses inward into the faster moving inner part, the elution curve will deviate from the kinematic limit, giving rise to a point of inflection or a shoulder in the curve. The emergence of the shoulder is to shorten the tailing; the concentration drops to zero at a much faster rate than the kinematic limit. At a farther distance from the injection point, the shoulder part of the curve becomes more developed; it becomes a rounded second peak that is comparable in height to the sharp first peak; see Fig. 7b, c. At a sufficiently long distance from the injection point, the sharp initial rise dwindles to be gradually replaced by a gentle rise, while the rounded second peak is fully 
developed to become the center peak, arriving at a time given by the distance divided by the mean velocity; see Fig. 7d. This is the onset of the Taylor-Aris regime, as the axial distribution becomes increasingly symmetrical about its peak approaching a Gaussian distribution. The limiting steady state results from an equilibrium interaction between convection and radial diffusion.

When there is slip on the wall, the following effects on the breakthrough/elution curves can be observed. First, the arrival of the sharp initial rise is delayed. This is because the centerline velocity $\hat{u}(0)=2(1+2 \hat{\lambda}) /(1+4 \hat{\lambda})$ is a decreasing function of the slip length. Second, the tailing is much shortened or even practically non-existent for moderately large slip. This follows from the fact that, owing to the wall slip, the solute does not stick to the wall and the entire solute cloud can be eluted within a finite time even in the kinematic limit. For slip length $\hat{\lambda}=0.5$, the decline of the curve remains sharp all the time. Third, the second peak is developed faster, and as a result the Taylor-Aris limit is approached earlier than the no-slip case. For slip length $\hat{\lambda}=0.5$, the distribution is already nearly Gaussian by a distance of $\hat{z}=0.5$. As an overall consequence, and also being the most important effect, the rate of spread of the profile is decreased by the slip. The larger the slip length, the smaller the spread of the elution curve at the same axial location.

Let us now look into the moments of the RTD that compactly describe an elution profile (Adrover et al. 2009):

$m^{(n)}=\frac{\int_{0}^{\infty} \bar{t}^{n} \overline{\hat{C}} \mathrm{~d} \hat{t}}{\int_{0}^{\infty} \overline{\hat{C}} \mathrm{~d} \hat{t}}$.

The first moment $m^{(1)}=\hat{t}_{c}$ corresponds to the mean residence time, while the second moment gives information about the dispersion. The second order moment about the center of profile $\sigma^{2}=m^{(2)}-\left(m^{(1)}\right)^{2}$ is the variance of the distribution $\overline{\hat{C}}(\hat{t})$ about $\hat{t}=\hat{t}_{c}$, the rate of change of which reflects the dispersion. The mean residence time and the variance are plotted in Fig. 8a, b as functions of the axial position for the three values of slip length. On the one hand, the mean residence time is only slightly affected by the slip. The $\hat{t}_{c}$ versus $\hat{z}$ curve becomes more like a straight line with a unity slope for larger $\hat{\lambda}$. Hence, under slip condition, the solute cloud as a whole tends to move at the mean flow velocity at all times, short or long. In contrast,
Fig. 8 a The mean residence time $\hat{t}_{c}, \mathbf{b}$ the variance $\sigma^{2}$ and $\mathbf{c}$ the normalized variance $\sigma^{2} / \hat{t}_{c}^{2}$ of the elution profiles, as functions of the axial position $\hat{z}$ for the slip length $\hat{\lambda}=0,0.05,0.5$ (a)

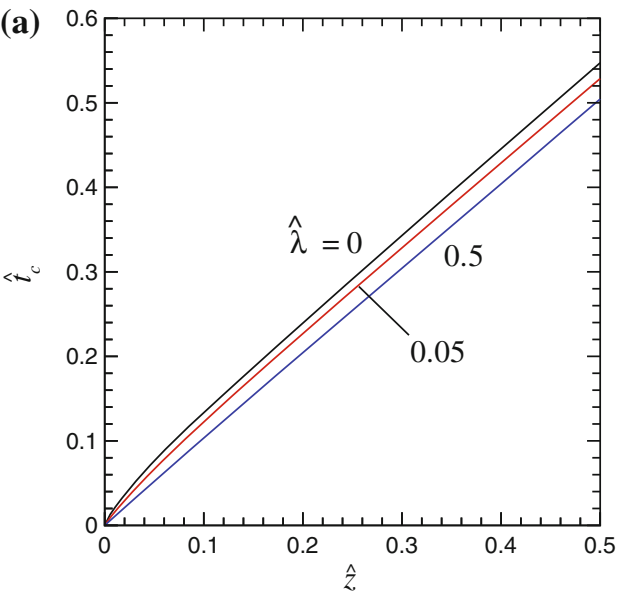

(c)

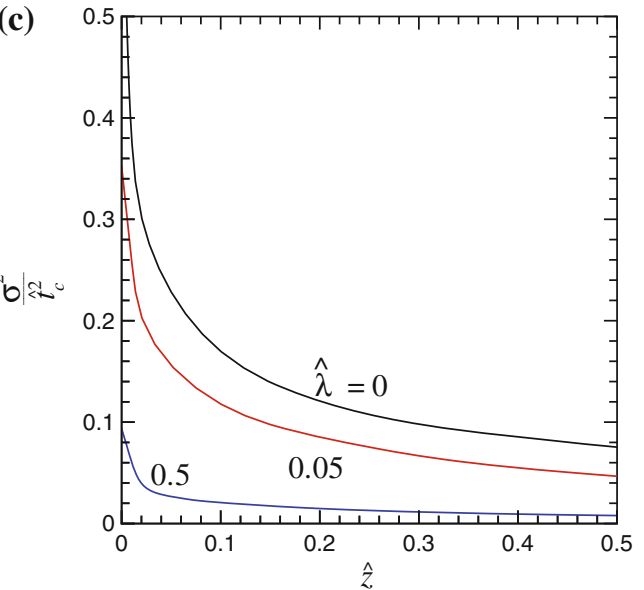

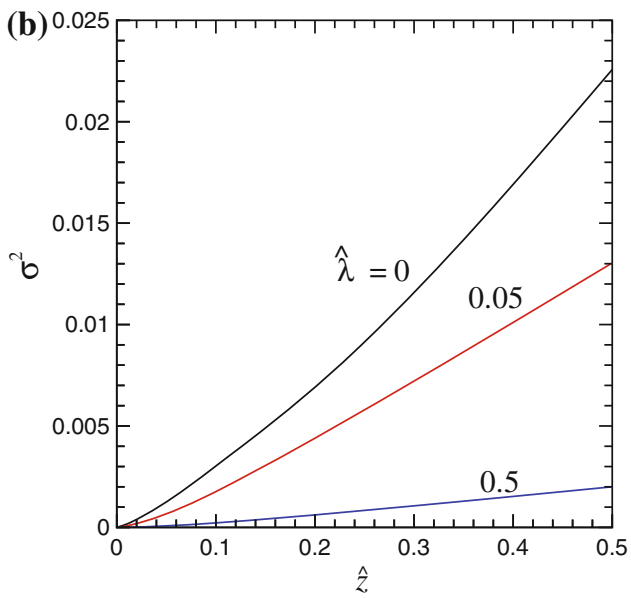


the movement of the cloud under no-slip condition is initially slower than the mean velocity, owing to the more extensive re-organization of the solute distribution taking place at small times. On the other hand, increasing the slip length will dramatically decrease the variance. For slip length $\hat{\lambda}=0.5$, the variance is less than one-tenth that of the no-slip case by an axial distance $\hat{z}=0.5$. The decreasing effect of slip on dispersion is conspicuously shown in Fig. 8c, where the normalized variance $\sigma^{2} / \hat{t}_{c}^{2}$ (equal to twice the vessel dispersion number, as discussed earlier) is seen to drop to virtually zero for slip length $\hat{\lambda}=0.5$ at an axial distance $\hat{z}=0.5$.

Following Adrover et al. (2009), let us further examine how in the convection-dominated regime the mean residence time and variance of the profiles may depend on the slip length. Adrover et al. (2009) have shown that, when the effective Péclet number $\mathrm{Pe}_{\mathrm{eff}}=\bar{u} a^{2} / z D=\hat{z}^{-1}$ is as large as $O\left(10^{3}\right)-O\left(10^{7}\right)$, the mean residence time and the variance in the slip cases will be saturated: they do not change with the effective Péclet number anymore, unlike the no-slip case. This distinguishing feature can be employed to detect whether the flow has any slip. Here, we show in Fig. 9, for very short distances, the mean residence time and variance (which are normalized with respect to $\hat{z}$ and $\hat{z}^{2}$, respectively, in order to reveal the saturation effect, if any) as functions of the reciprocal of the axial distance $\hat{z}^{-1}$. The distance being considered is as short as 0.0035 . The finite initial slug length and also the spatial discretization prevented us from considering a too small axial length here. Therefore, we have considered $\hat{z}^{-1}$ only being as large as $O\left(10^{2}\right)$. We find that, over this relatively small order of the effective Péclet number, the normalized mean residence time, $\hat{t}_{c} / \hat{z}$, will already tend to a constant in the slip cases. It appears that the saturation begins at smaller $\hat{z}^{-1}$ for larger slip length. In contrast, the normalized variance, $\sigma^{2} / \hat{z}^{2}$, of the slip cases does not exhibit any sign of saturation over this range of the effective Péclet number. One may need to consider much larger $\hat{z}^{-1}$ before the saturation can be seen for the variance. The results suggest that if slip is to be inferred from this indirect transportbased method, one should perhaps pay more attention to the first moment than the second moment if the effective Péclet number is only moderately large.

\section{Concluding remarks}

Dispersion coefficients have been deduced for Taylor-Aris dispersion in Poiseuille flow through plane and circular channels under the influence of wall slippage. The time development of convection-dominated dispersion in slip flow through a circular channel has also been investigated numerically. For comparison with the no-slip case, we have separately considered the cases when either the pressure gradient or the flow rate is kept fixed while changing the slip length. In a channel with a constant slip length, the spreading of a solute cloud is in general diminished by the boundary slip in the following manners. If under a fixed pressure gradient, the dispersivity remains the same, but the convection speed is increased when compared with the no-slip case. Therefore, because of a shorter travel time, the spread of the cloud on reaching the channel outlet is smaller than the no-slip counterpart. If under a fixed flow rate, the convection speed is not changed, but the dispersivity decreases owing to a flattened velocity profile. This will of course reduce the spread of the cloud as well.

The decreasing effect of slip is, however, adversely affected by (i) disparity in the slip lengths of the walls, and (ii) phase exchange with the wall. In our problem of flow through a plane channel, we have found that a large
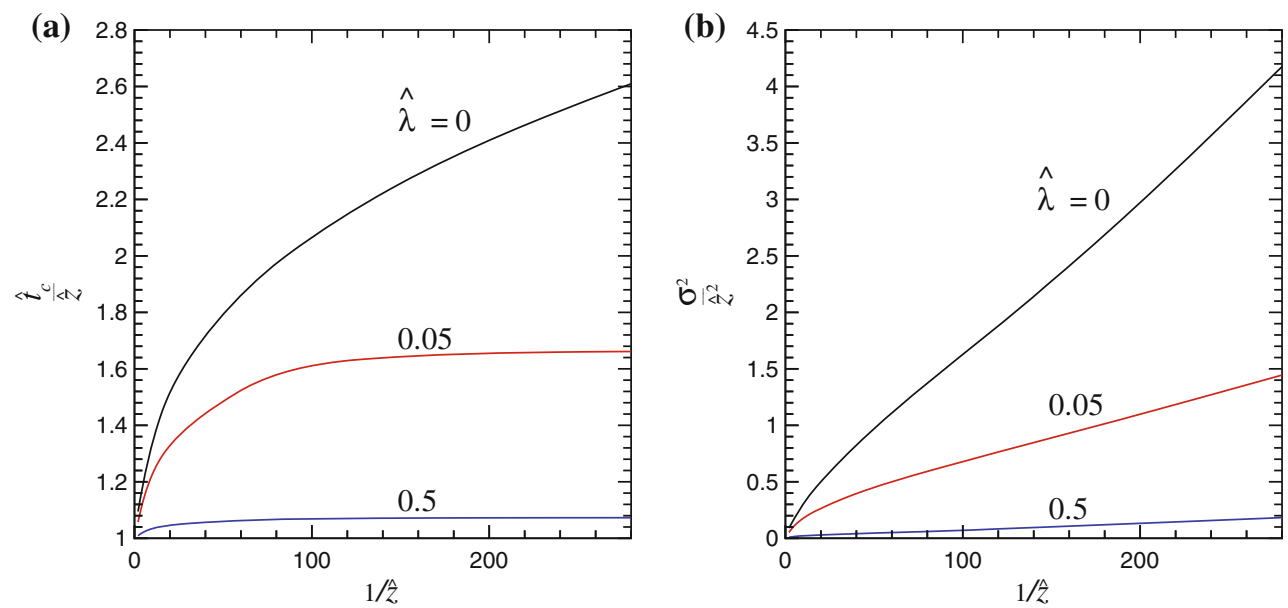

Fig. 9 a The mean residence time $\hat{t}_{c}$ normalized by $\hat{z}$, and $\mathbf{b}$ the variance $\sigma^{2}$ normalized by $\hat{z}^{2}$ of the elution profiles, as functions of the reciprocal of the axial position $\hat{z}^{-1}$ for the slip length $\hat{\lambda}=0,0.05,0.5$ 
disparity in the slip lengths of the two walls has the effect of increasing the dispersivity, as a result of increasing the skewness of the velocity profile. In our problem of flow through a circular channel, we have also found that even a small degree of phase exchange with the wall can materially diminish or even reverse the decreasing effect of slip on dispersion.

One distinguishing quality of transport in slip flow shows up in the pure convection kinematic limit. Without diffusion, the slip alone enables a solute cloud to be completely eluted out of a channel in a finite time. This is in sharp contrast to the no-slip case, in which the elution time is in theory infinitely long, if diffusion is absent. We have followed a recent study in the literature, and looked into how such a distinguishing feature is manifested in the saturation of the moments of the elution profiles at short distances from the injection point. Our results suggest that the saturation occurs to the mean residence time at lower (also more practical) effective Péclet number than the variance.

The present study is limited to walls with constant slip lengths. It is worth extending the study to heterogeneous walls with, say, patterned slippage (Hendy et al. 2005), for which the flow is periodic and has transverse components that may lead to nontrivial effects on the solute dispersion.

Acknowledgments The study was supported by the Research Grants Council of the Hong Kong Special Administrative Region, China, through Project No. HKU 715609E, and also by the University of Hong Kong through the Small Project Funding Scheme under Project Code 200807176081. Useful comments by the reviewers are gratefully acknowledged.

Open Access This article is distributed under the terms of the Creative Commons Attribution Noncommercial License which permits any noncommercial use, distribution, and reproduction in any medium, provided the original author(s) and source are credited.

\section{References}

Adrover A, Cerbelli S, Garofalo F, Giona M (2009) Convectiondominated dispersion regime in wide-bore chromatography: a transport-based approach to assess the occurrence of slip flows in microchannels. Anal Chem 81:8009-8014

Ajdari A, Bontoux N, Stone HA (2006) Hydrodynamic dispersion in shallow microchannels: the effect of cross-sectional shape. Anal Chem 78:387-392

Aris R (1959) On the dispersion of a solute by diffusion, convection and exchange between phases. Proc R Soc Lond A 252:538-550

Beard DA (2001) Taylor dispersion of a solute in a microfluidic channel. J Appl Phys 89:4667-4669

Boris JP, Landsberg AM, Oran ES, Gardner JH (1993) LCPFCT-a flux-corrected transport algorithm for solving generalized continuity equations. Laboratory for Computational Physics and Fluid Dynamics, Naval Research Laboratory, Washington, DC
Cheng YP, Teo CJ, Khoo BC (2009) Microchannel flows with superhydrophobic surfaces: effects of Reynolds number and pattern width to channel height ratio. Phys Fluids 21:122004

Choi CH, Ulmanella U, Kim J, Ho CM, Kim CJ (2006) Effective slip and friction reduction in nanograted superhydrophobic microchannels. Phys Fluids 18:087105

Cottin-Bizonne C, Barentin C, Charlaix E, Bocquet L, Barrat JL (2004) Dynamics of simple liquids at heterogeneous surfaces: molecular-dynamics simulations and hydrodynamic description. Eur Phys J E 15:427-438

Dutta D, Ramachandran A, Leighton DT Jr (2006) Effect of channel geometry on solute dispersion in pressure-driven microfluidic systems. Microfluid Nanofluid 2:275-290

Golay MJE, Atwood JG (1979) Early phases of the dispersion of a sample injected in Poiseuille flow. J Chromatogr 186:353-370

Hendy SC, Jasperse M, Burnell J (2005) Effect of patterned slip on micro- and nanofluidic flows. Phys Rev E 72:016303

Kalweit M, Drikakis D (2008) Multiscale methods for micro/nano flows and materials. J Comput Theor Nanosci 5:1923-1938

Kreutzer MT, Günther A, Jensen KF (2008) Sample dispersion for segmented flow in microchannels with rectangular cross section. Anal Chem 80:1558-1567

Lauga E, Brenner MP, Stone HA (2007) Microfluidics: the no-slip boundary condition. In: Foss J et al. (eds) Springer handbook of experimental fluid mechanics. Springer, Heidelberg, pp 12191240

Lee C, Choi CH, Kim CJ (2008) Structured surfaces for a giant liquid slip. Phys Rev Lett 101:064501

Levenspiel O (1999) Chemical reaction engineering, 3rd edn. Wiley, New York

Mayock KP, Tarbell JM, Duda JL (1980) Numerical simulation of solute dispersion in laminar tube flow. Sep Sci Technol 15:12851296

Mei CC, Auriault JL, Ng CO (1996) Some applications of the homogenization theory. Adv Appl Mech 32:277-348

$\mathrm{Ng}$ CO (2000) A note on the Aris dispersion in a tube with phase exchange and reaction. Int J Eng Sci 38:1639-1649

$\mathrm{Ng}$ CO (2006) Dispersion in steady and oscillatory flows through a tube with reversible and irreversible wall reactions. Proc $\mathrm{R}$ Soc A $462: 481-515$

Ng CO, Rudraiah N (2008) Convective diffusion in steady flow through a tube with a retentive and absorptive wall. Phys Fluids 20:073604

$\mathrm{Ng}$ CO, Yip TL (2001) Effects of kinetic sorptive exchange on solute transport in open-channel flow. J Fluid Mech 446:321-345

Rothstein JP (2010) Slip on superhydrophobic surfaces. Annu Rev Fluid Mech 42:89-109

Rush BM, Dorfman KD, Brenner H (2002) Dispersion by pressuredriven flow in serpentine microfluidic channels. Ind Eng Chem Res 41:4652-4662

Stone HA, Stroock AD, Ajdari A (2004) Engineering flows in small devices: microfluidics toward a lab-on-a-chip. Annu Rev Fluid Mech 36:381-411

Taylor GI (1953) Dispersion of soluble matter in solvent flowing slowly through a tube. Proc R Soc Lond A 219:186-203

Vikhansky A (2009) Taylor dispersion in shallow micro-channels: aspect ratio effect. Microfluid Nanofluid 7:91-95

Wooding RA (1960) Instability of a viscous liquid of variable density in a vertical Hele-Shaw cell. J Fluid Mech 7:501-515

Wu Z, Nguyen NT (2005) Convective-diffusive transport in parallel lamination micromixers. Microfluid Nanofluid 1:208-217 